\title{
COVID-19 in perspective
}

\section{Introduction}

The world is in the midst of a COVID-19 global pandemic and public health crisis which has resulted in considerable morbidity and mortality and placed significant stress on healthcare resources. The need to dedicate major economic, infrastructural and medical resources to critically ill COVID-19 patients has resulted in a redistribution of the activities of medical disciplines not primarily involved in the management of COVID-19 patients. Given the varying nature of international healthcare systems, conditions differ significantly by region and locality and between state and private practice. Critical decisions concerning the deployment of resources and the management of elective surgical procedures are based on institutional policies and recommendations from local, regional and national authorities, and specialist societies and must consider the availability of finite and essential resources.

The key principles of management are to maintain essential care while minimising risks of COVID-19 to patients and staff and maintaining overall healthcare resources. As the COVID-19 pandemic evolves, surgical procedures will be increased or reduced based on the current level of restriction to healthcare services. Although some consistency across the country is desirable, provincial and regional considerations will of necessity influence how these recommendations are implemented. Healthcare professionals have a responsibility to maximise the use of these resources to provide the best possible care for all patients. Although elective surgical procedures are being postponed and cancelled in many parts of the world during the COVID-19 pandemic, acute procedures will continue and will need to be appropriately managed.

There is currently a paucity of information to delineate the principles of ethics for the management of elective and emergent surgery lists during the COVID-19 pandemic. Most routine problems are appropriately managed within the context of surgical practice at individual level and/or in the context of a multidisciplinary team; health professionals may also consult their peers for advice or look to existing policies on ethical issues, and follow their professional lead body.

Boundaries of responsibility in relation to who has access to new technological treatments, and who decides when to withhold or withdraw treatment, for example, have challenged long-held paternalistic views about how surgery should be practised. Decision-making gradually ceased to be the responsibility of one individual, the specialist in charge, and has become a shared responsibility, involving patients, their families, and members of the multidisciplinary team. In addition, where complex legal and ethics decisions have to be made, the role played by specially formed ethics committees has received recognition.

All surgical disciplines are involved in the pandemic and most areas are affected. This collection of opinion pieces highlights and addresses COVID-19 relevant issues pertaining to ethical considerations, enhanced recovery after surgery, ENT, breast reconstruction and transplantation. These views are those of the authors and not position statements of national societies or governmental departments. As such we hope they will encourage collegial debate on the subject as we move through the phases of the pandemic.

\section{SR Thomson, ${ }^{1}$ JEJ Krige ${ }^{2}$}

${ }^{1}$ Editor, South African Journal of Surgery

${ }^{2}$ Editor Emeritus, South African Journal of Surgery 